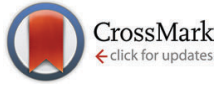

Cite this: J. Mater. Chem. C, 2015, 3, 3908

Received 8th February 2015, Accepted 4th March 2015

DOI: $10.1039 / \mathrm{c} 5 \mathrm{tc00380f}$

www.rsc.org/MaterialsC

\title{
Silver nanowire coated threads for electrically conductive textiles
}

\author{
Yahya Atwa, Nupur Maheshwari and Irene A. Goldthorpe*
}

\begin{abstract}
The emerging area of e-textiles requires electrically conductive threads. We demonstrate that nylon, polyester, and cotton threads can be made conductive by coating their surfaces with random networks of solution-synthesized silver nanowires. A resistance per unit length of $0.8 \Omega \mathrm{cm}^{-1}$ was achieved and can be varied through the density of the nanowire coating. Because the nanowires are $35 \mathrm{~nm}$ in diameter, and the mesh structure does not cover the entire surface like a thin-film, less metal is used compared to conventional silver-coated conductive threads. This leads to a much lower weight and mechanically flexible coating. The functionality of the thread as a heater and the fabrication of stretchable conductive thread are also demonstrated.
\end{abstract}

\section{Introduction}

In recent years a new research direction in the area of electronic textiles has emerged. ${ }^{1}$ In this growing field, electrically conductive thread is used for electromagnetic interference (EMI) shielding ${ }^{2}$ and wearable antennas, ${ }^{3}$ as well as functions as interconnects in textile-integrated electronic devices such as health monitoring sensors ${ }^{4}$ and solar cells. ${ }^{5}$ Commonly used textiles such as cotton, nylon, and polyester are all electrical insulators. Commercially available conductive thread is typically either a solid metal wire, such as copper or stainless steel, or a non-conductive thread coated with a $\sim 1 \mu \mathrm{m}$ thick metal film, usually silver. These options are less than ideal as they tend to be stiff and brittle, which causes problems both during weaving and in end applications. Furthermore, they can breakdown (resistance greatly increases) after repeated bends. ${ }^{6}$

Alternative coatings to achieve flexible conductive threads have recently been proposed such as conductive polymers, ${ }^{7}$ graphene flakes, ${ }^{8}$ and carbon nanotubes. ${ }^{9}$ One of the main problems of conducting polymers is that they are unstable in air because of the absorption of oxygen and moisture. ${ }^{10}$ Additionally, the conductivity of polymer-coated thread is low ${ }^{11}$ and therefore cannot be used in many applications. Regarding carbon nanotubes, typically multi-walled tubes are used because they are metallic. However, although the resistance of individual carbon nanotubes (or graphene flakes) is extremely low, the junction resistance between two overlapping nanotubes (or flakes) in the film is very high. ${ }^{12,13}$ This leads to thread resistances per unit length on the order of $1 \mathrm{k} \Omega \mathrm{cm}^{-1} \cdot{ }^{14}$ Carbon nanotubes and graphene can have stability issues in air as well, ${ }^{15}$ and evidence

Department of Electrical \& Computer Engineering, University of Waterloo, Waterloo, ON, Canada N2L 3G1. E-mail: igoldthorpe@uwaterloo.ca exists that carbon nanotubes may be toxic upon skin exposure. ${ }^{16}$ Achieving conductive thread by coating with metallic nanoparticles such as gold, silver, or platinum has also been demonstrated. ${ }^{17-19}$ Of these, silver is the most common because it is the most conductive of all metals, is more cost effective than gold or platinum, and is relatively stable in air. However, the conductivity of metallic-nanoparticle coated threads is low because their small size leads to many inter-particle junctions.

In this work, nylon, polyester, and cotton threads are instead coated with a thin metallic mesh made up of a random network of silver nanowires. The use of metallic nanowires in textiles has only been briefly mentioned in the literature ${ }^{20,21}$ with little accompanying data. Unlike silver nanoparticles, the elongated shape of a nanowire allows for a conductive film to be achieved at a far lower particle density and therefore there are less junctions. And unlike carbon-based materials, metal junctions can be sintered to greatly reduce junction resistance. Because the nanowire coating is a mesh rather than a continuous film, and the nanowires used are only $35 \mathrm{~nm}$ thick, much less metal is used compared to conventional conductive threads where a metal film coating or a solid wire is used. This can lead to a lower material cost, lower weight, and thinner thread, as well as greater mechanical flexibility. The coating can be simply deposited as a dye with no vacuum or complex processes required.

\section{Experimental methods}

\subsection{Coating procedure}

Silver nanowires can be synthesized in solution at relatively low temperatures by the now well-known polyol method. ${ }^{22}$ In a common implementation of this method, a mixture of $\mathrm{AgNO}_{3}$ and ethylene glycol (EG) is regularly added to a solution containing EG with polyvinylpyrrolidone (PVP) and $\mathrm{NaCl}$ and is heated 


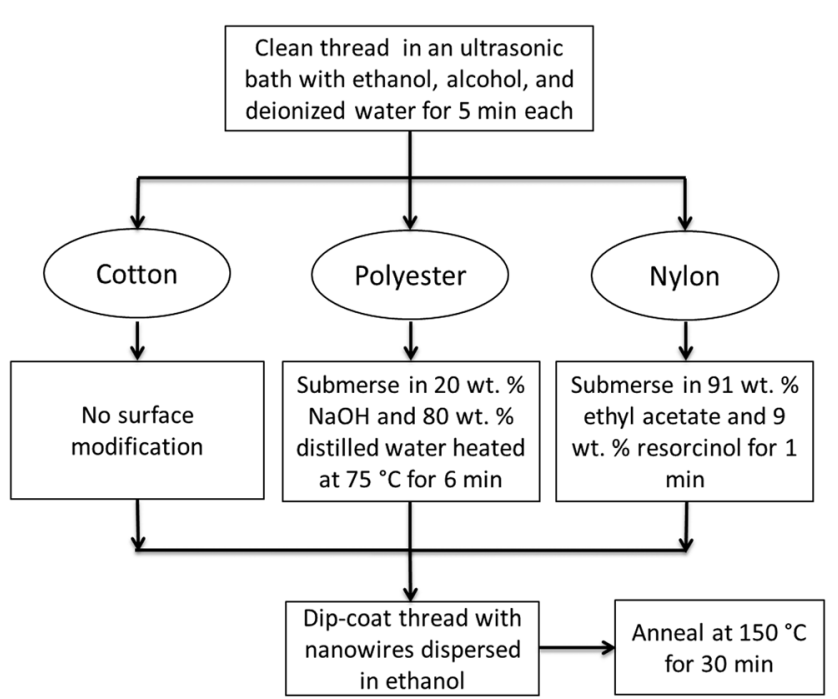

Fig. 1 Coating procedure of the conductive thread.

to $170{ }^{\circ} \mathrm{C}$. The resulting nanowires are crystalline with five twin planes running along the longitudinal axis. ${ }^{22}$ The silver nanowires used in this work were supplied in ethanol from Blue Nano Inc (Charlotte, North Carolina). Their average diameters and lengths were $35 \mathrm{~nm}$ and $15 \mu \mathrm{m}$, respectively.

Deposition of nanowire films around threads was achieved through dip-coating. The procedure is illustrated in Fig. 1. The cotton and polyester threads were multifilament with diameters of $200 \mu \mathrm{m}$ and $300 \mu \mathrm{m}$, respectively. The nylon thread was monofilament with a diameter of $700 \mu \mathrm{m}$. All types of thread were cleaned in an ultrasonic bath using ethanol, alcohol, and deionized water for $5 \mathrm{~min}$ each. The nanowire solution adhered well to the surface of the cotton thread, but not to polyester and nylon. These latter two synthetic textiles required a chemical pre-treatment. After cleaning, the polyester threads were submersed for 6 minutes in a solution consisting of $20 \mathrm{wt} \% \mathrm{NaOH}$ and $80 \mathrm{wt} \%$ distilled water heated at $75{ }^{\circ} \mathrm{C}$, then dried in hot air. The nylon threads were submersed in a solution consisting of $91 \mathrm{wt} \%$ ethyl acetate and $9 \mathrm{wt} \%$ resorcinol for 1 minute and then dried in air. In all the cotton, polyester and nylon cases, the density of the deposited nanowire film was varied through the concentration of the nanowires in the coating solution and through the number of dipping steps. After deposition the threads were annealed in air at $150{ }^{\circ} \mathrm{C}$ for $30 \mathrm{~min}$.

To obtain stretchable conductive thread, a multifilament polyester/rubber blend ( $28 \%$ polyester, $72 \%$ rubber) with a diameter of $450 \mu \mathrm{m}$ was coated. The thread was stretched to $150 \%$ of its original length using a vice, surface modified with $\mathrm{NaOH}$, and then coated with silver nanowires in the stretched state by drop-casting. The coated-thread was then annealed using a heat gun at $150{ }^{\circ} \mathrm{C}$ for $60 \mathrm{~min}$.

\subsection{Characterization and measurement}

To measure resistivity, copper tape was affixed to each end of the thread and connected to a multimeter using alligator clips. As is typical for conductive threads, the resistance of the threads will be stated in resistance per unit length of thread, or $\Omega \mathrm{cm}^{-1}$.
The current-voltage relationship was obtained using the multimeter and a direct current (DC) power supply.

The density of nanowires in the coating was calculated from scanning electron microscopy (SEM) images and imaging software. Flexibility was measured by bending the thread around a rod with a $6 \mathrm{~mm}$ radius and measuring the resistance after successive bends. To investigate washability, a solution of commercially available detergent dissolved in water was heated to $25{ }^{\circ} \mathrm{C}$ and stirred with a magnetic stir bar rotating at $500 \mathrm{rpm}$. The threads were repeatedly immersed in this solution and their resistance was measured after 5 minute intervals. To investigate the ability of the thread to act as a heater, the DC power supply was used to pass a current and the thread surface temperature was measured using a thermocouple. The performance of the stretchable thread was assessed by monitoring its resistance during 10 stretch-relapse cycles in the vice.

For comparison purposes, flexibility and washing tests were also performed on a commercially available conductive thread (117/17 2-ply manufactured by Shieldex-U.S., Palmyra, NY). This commercial thread is multifilament nylon, where the surface of each filament is coated with a thin-film of silver.

\section{Results and discussion}

\subsection{Microstructure and electrical characteristics}

Silver nanowires synthesized by the polyol process as described above have a 1-3 nm thick PVP layer on their sidewalls. ${ }^{22}$ This polymer coating is hydrophilic. Because cotton is also hydrophilic, surface modification was not required for nanowire adhesion. Polyester and nylon, on the other hand, are hydrophobic. Dipping polyester thread in the nanowire solution resulted in a resistance of $>300 \Omega \mathrm{cm}^{-1}$ (post-annealing) and the nylon thread did not have any measureable conductivity. $\mathrm{NaOH}$ is commonly used in the textile industry for scouring, which removes impurities, and is also known to render polyester more hydrophilic. ${ }^{23}$ The $\mathrm{NaOH}$ creates polar hydroxyl groups along the polyester chains which can then form hydrogen bonds with the carbonyl groups of PVP, thus resulting in nanowire adhesion. Similarly, resorcinol creates polar hydroxyl groups on the surface of nylon.

SEM images of nanowire-coated nylon, polyester and cotton threads are displayed in Fig. 2. The nanowires form a connected network that extends completely around the thread diameter and along the length. However, without an annealing step the resistance of the nanowire coating is very high because overlapping nanowires do not make good physical contact with one another, and the PVP layer on the nanowire surfaces is an insulator. ${ }^{24}$ Annealing both partially decomposes the PVP and fuses the nanowire junctions through sintering. The ability to sinter and greatly reduce junction resistance is a strong advantage of silver nanowires over carbon nanotubes. In this study it was found that annealing at $150{ }^{\circ} \mathrm{C}$ for between $30-60$ minutes yielded the lowest thread resistance. For annealing times longer than 60 minutes, or at temperatures above $150{ }^{\circ} \mathrm{C}$, the resistance was higher due to the melting of the nanowires into disconnected segments. ${ }^{25}$ 


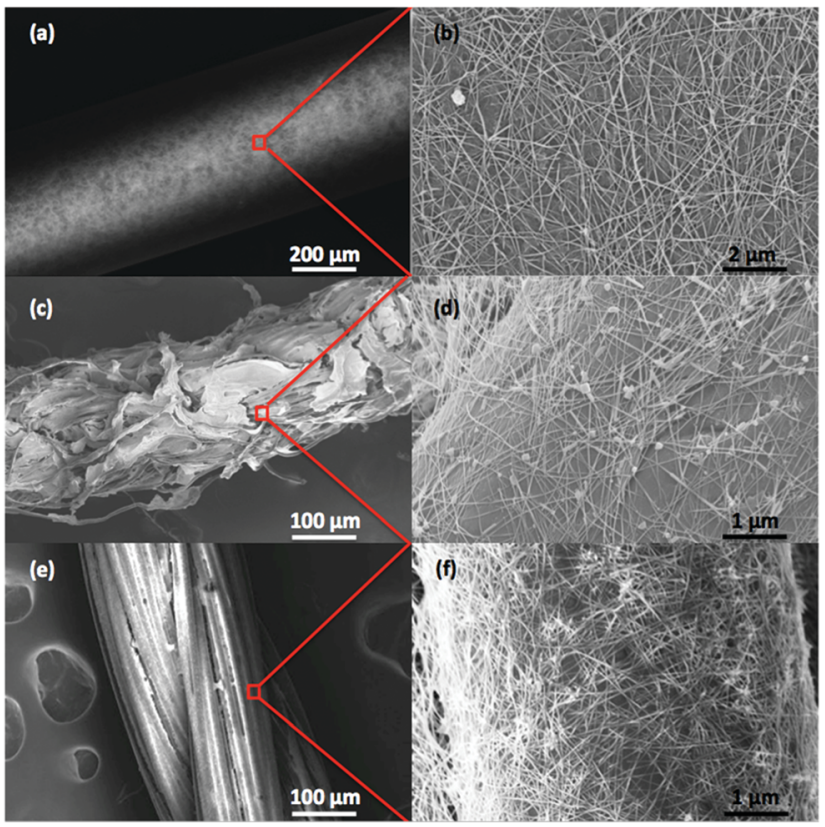

Fig. 2 SEM images of silver-nanowire-coated (a, b) nylon thread, (c, d) cotton thread and $(e, f)$ polyester thread.

In the SEM images, the nylon thread (Fig. 2a and b) was dipped 3 times in a solution containing $1.25 \mathrm{mg} \mathrm{mL}^{-1}$ of silver nanowires in ethanol and has a resistance of $12 \Omega \mathrm{cm}^{-1}$. The cotton thread (Fig. 2c and d) was dipped 3 times in a nanowire solution with a concentration of $5 \mathrm{mg} \mathrm{mL}^{-1}$ and has a resistance of $11 \Omega \mathrm{cm}^{-1}$. The polyester thread (Fig. 2e and f) was dipped 3 times in a nanowire solution with a concentration of $5 \mathrm{mg} \mathrm{mL}^{-1}$ and has a resistance of $15 \Omega \mathrm{cm}^{-1}$. In all cases, as the density of nanowires is increased, resistance decreases. Fig. 3a shows the $I-V$ relationship of nylon thread coated with three different nanowire densities. The curves are linear and thus conduction behaves like a metal. In Fig. 3b, nanowire-coated thread is used in a circuit to power an LED.

The dependence of resistance on density, as well the material costs of the nanowires, ${ }^{26}$ are tabulated in Table 1 for the nylon threads. A resistance as low as $0.8 \Omega \mathrm{cm}^{-1}$ was achieved. $0.8 \Omega \mathrm{cm}^{-1}$ in this case is equivalent to a sheet resistance of $0.18 \Omega$ square $^{-1}$, and the conductivity of the composite (using the cross-sectional area of the nylon thread) is $81 \mathrm{~S} \mathrm{~cm}^{-1}$. For comparison, nylon thread coated with the conductive polymer PEDOT:PSS has been reported to be $40 \Omega \mathrm{cm}^{-1},{ }^{27}$ and nylon thread coated with carbon nanotubes has been reported to be $49 \mathrm{k} \Omega \mathrm{cm}^{-1}$. $^{14}$

Although silver is an expensive material, so little of it is used so costs are low (Table 1). Furthermore, in regards to weight, around $1 \mathrm{mg} \mathrm{m}^{-1}$ or less is added to the thread. If the coating were instead a typical $1 \mu \mathrm{m}$ thick silver film the coating would weigh $23 \mathrm{mg} \mathrm{m}^{-1}$, so the nanowire coating is less than $5 \%$ the weight of a solid film.

\subsection{Mechanical properties}

Conductive thread must be mechanically flexible and its resistance must not degrade significantly after repeated bends, especially
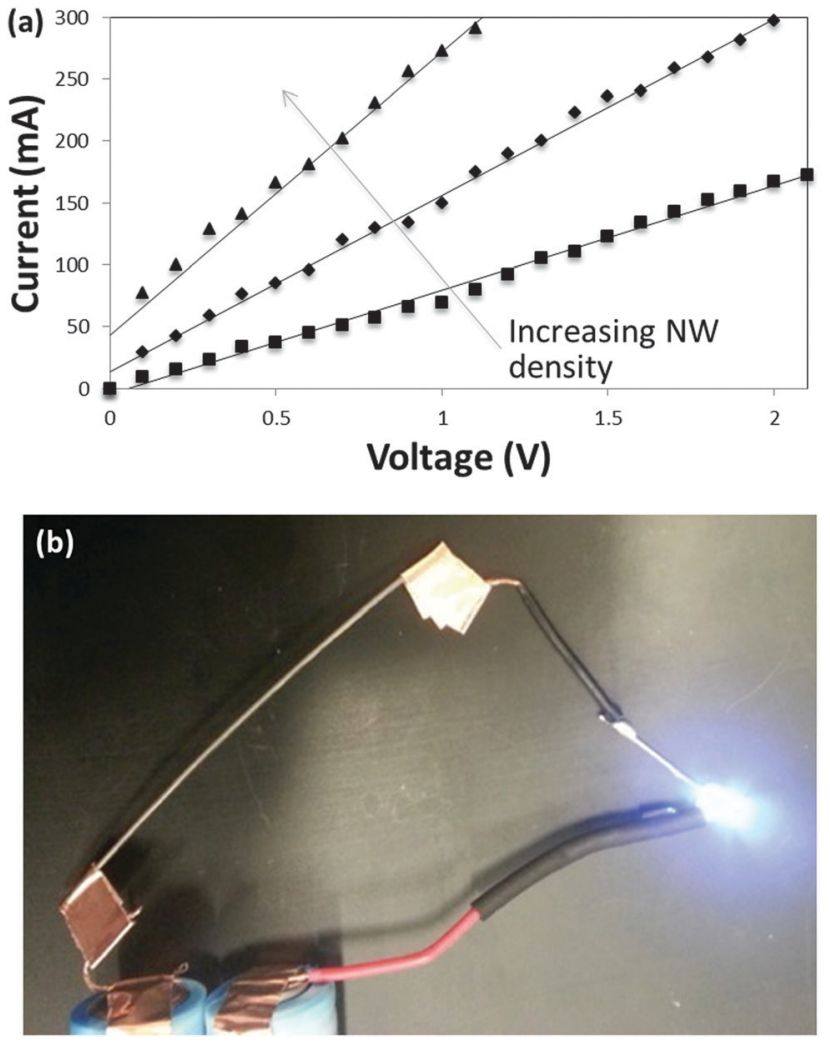

Fig. 3 (a) Current-voltage curves of nylon thread coated with different nanowire densities. (b) Passing current through nanowire-coated nylon thread (top left of image) to power an LED.

Table 1 The dependence of resistance and material cost on the coating density of the nanowires on nylon thread

\begin{tabular}{lll}
\hline $\begin{array}{l}\text { Metal } \\
\text { density }\left(\mathrm{mg} \mathrm{m}^{-1}\right)\end{array}$ & $\begin{array}{l}\text { Resistance per } \\
\text { length }\left(\Omega \mathrm{cm}^{-1}\right)\end{array}$ & $\begin{array}{l}\text { Nanowire } \\
\text { cost }\left(\$ \mathrm{~m}^{-1}\right)\end{array}$ \\
\hline 0.24 & 12.0 & 0.008 \\
0.52 & 2.5 & 0.017 \\
1.07 & 0.8 & 0.035
\end{tabular}

when used in applications such as clothing-integrated sensors. The variation of resistance with repeated bending for both the commercial conductive thread and a nanowire-coated nylon thread with the same initial resistance is plotted in Fig. 4 a. The resistance of the commercial thread increased by more than 4 times, from $2.8 \Omega \mathrm{cm}^{-1}$ to $12.2 \Omega \mathrm{cm}^{-1}$ after being bent 200 times. Silver thin-films are brittle and can crack after repeated bends ${ }^{28}$ thus increasing its resistance. Films of silver nanowires, on the other hand, are much more flexible; $;^{29}$ the cylindrical geometry and nanosized diameter of nanowires is known to make them stronger and more flexible than their bulk-like counterparts, ${ }^{30}$ and they can endure higher elastic strains. ${ }^{31}$ The resistance of the nanowire-coated thread only increased $14 \%$ after 200 bends, from $2.8 \Omega \mathrm{cm}^{-1}$ to $3.2 \Omega \mathrm{cm}^{-1}$. Furthermore, when the resistance of nanowire-coated threads was measured when the thread was bent (as opposed to measuring the resistance after returning the thread flat as was done to collect the data in Fig. 4a), the resistance was actually lower than 

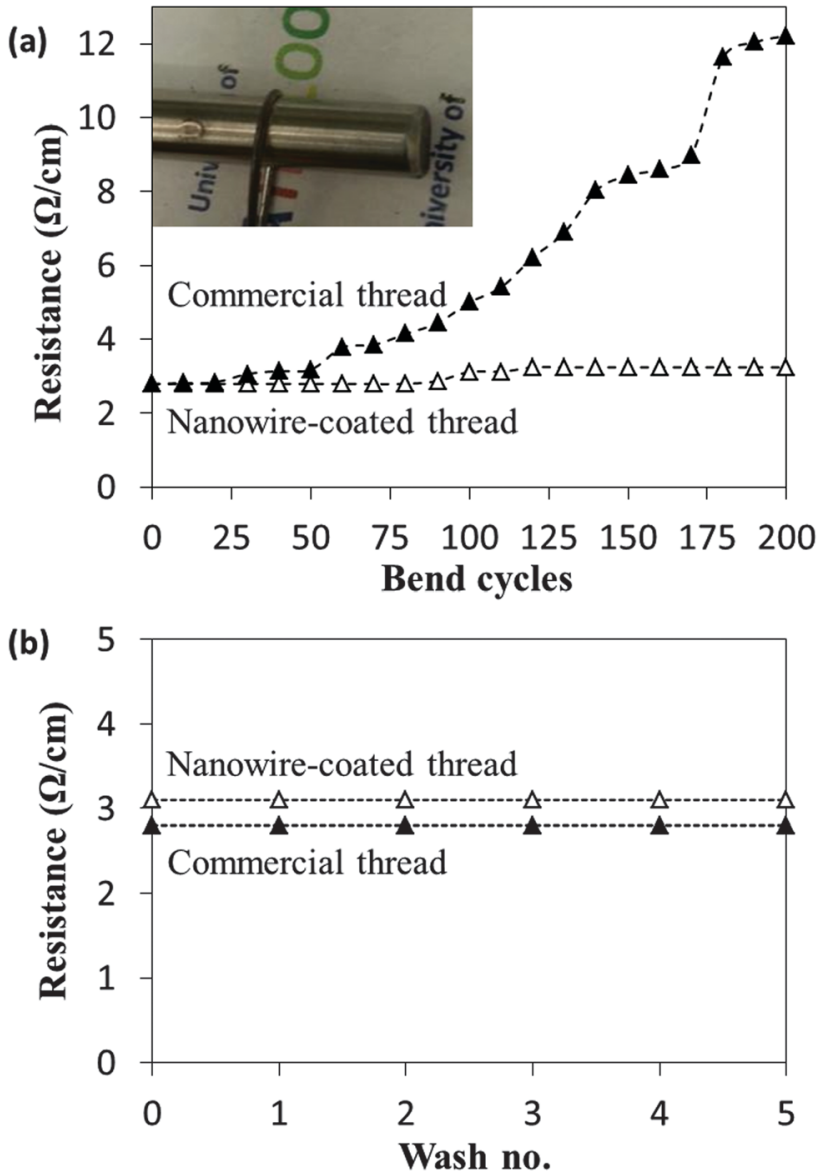

Fig. 4 The resistance of a nanowire-coated nylon thread and a commercially available conductive thread after (a) repeated bends to a $6 \mathrm{~mm}$ radius of curvature and (b) after repeated washing.

an unbent thread. For example, when an initially $2.8 \Omega \mathrm{cm}^{-1}$ nanowire-coated nylon thread was bent around a rod with a radius of $6 \mathrm{~mm}$, its resistance decreased to $2.3 \Omega \mathrm{cm}^{-1}$. This may be because bending causes mechanical forces that improve the connections between overlapping nanowires and thus lower their junction resistances. The resistance of the commercial thread, on the other hand, increased from $2.8 \Omega \mathrm{cm}^{-1}$ to $4.4 \Omega \mathrm{cm}^{-1}$ when bent.

\subsection{Washability}

Adhesion of the nanowire coating to the thread is another important parameter. As can be seen in Fig. $4 \mathrm{~b}$, the resistance of the nanowire-coated nylon thread did not change after five repeated washings in liquid detergent. This indicates that the coating did not come off or degrade and that the chemical pretreatment of the nylon provides good adhesion. The resistance of the commercial thread also maintained its conductivity after the same washing procedure.

\subsection{Thread heater}

Conductive threads can be used as a heating element, where electricity is converted to heat through Joule heating. Thread heaters can have applications in areas such as car seats, heated

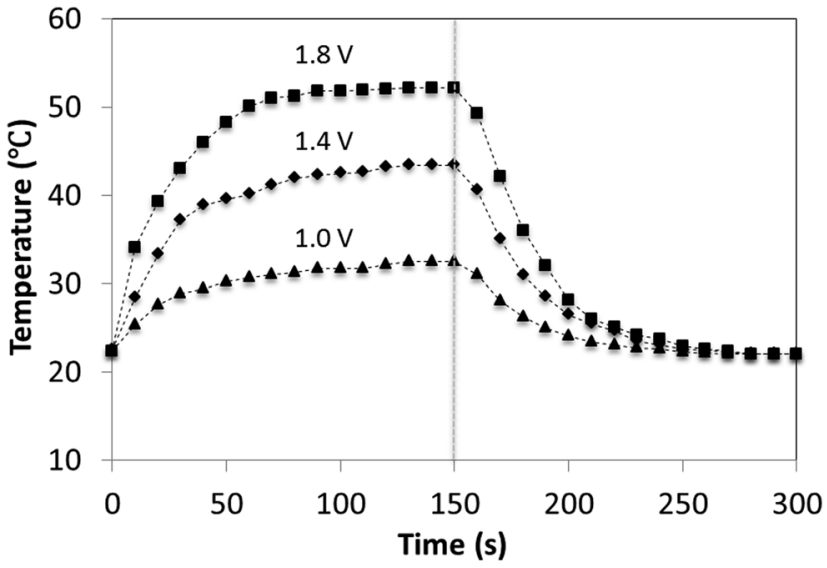

Fig. 5 Temperature profiles of the thread heater at different input voltages. Voltages were applied across a $1.4 \mathrm{~cm}$ long section of thread for $150 \mathrm{~s}$.

clothing and anti-freezing materials. The temperature response of a nanowire-coated nylon thread to three different applied voltages is plotted in Fig. 5. When a bias of $1.8 \mathrm{~V}$ is applied across a $1.4 \mathrm{~cm}$ long section of thread, a temperature above $50{ }^{\circ} \mathrm{C}$ is achieved, demonstrating its functionality at low voltages. Furthermore, for all magnitudes of voltage used, the steadystate temperature was achieved within $60 \mathrm{~s}$, confirming a fast response time of the thread heater.

\subsection{Stretchable conductive thread}

For certain e-textile applications involving clothing, sporting apparel, strain sensors, etc., elastic strechability is desired. However, few options for stretchable conductive thread exist; located options have resistances in the $\mathrm{k} \Omega \mathrm{cm}^{-1}$ range and have no data on the stability of resistance with stretching.

In Fig. 6, the resistance of the nanowire-coated polyester/ rubber thread as it is stretched and relapsed is plotted. After several stretching cycles, the resistance of the thread remains relatively constant with subsequent cycles. The inset of Fig. 6 is

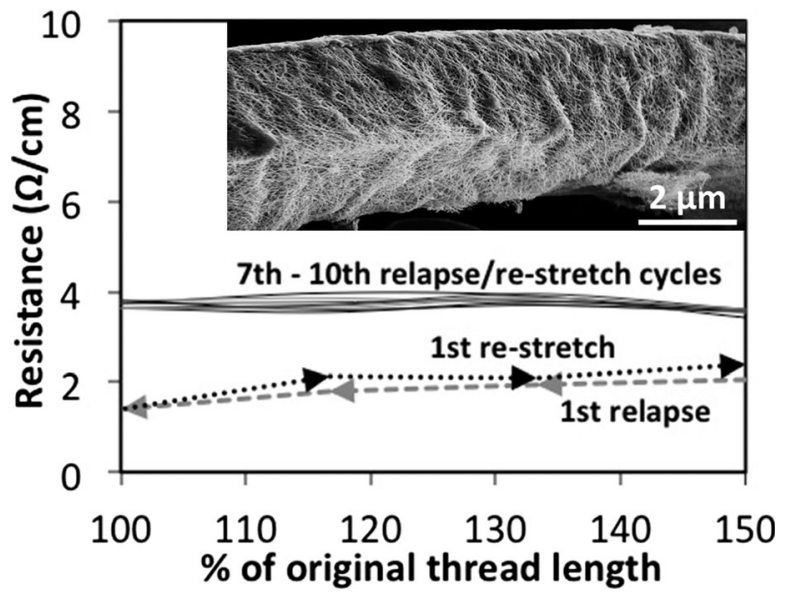

Fig. 6 Resistance of a nanowire-coated polyester/rubber thread during its first and seventh through tenth stretching cycles. Inset: SEM image of a filament in the thread after being stretched ten times and returned to its original length. 
an SEM image of one filament of the thread after being stretched 10 times and returned to its original length. A buckling of the nanowire coating can be seen. A flattening out and return to this wavy coating provides a mechanism for stable resistance with changing strain. A similar buckling strategy has been implemented for stretchable conductive planar thin-films. ${ }^{32}$ Coating of the polyester/rubber thread in an un-stretched state was also tried, but the resistance change with stretching was far higher than shown in Fig. 6 since buckling did not occur.

\section{Conclusions}

Cotton, polyester and nylon threads were successfully made electrically conductive by coating their surfaces with meshes of silver nanowires. The nanowire coating is less than $5 \%$ the weight of typical silver thin-film coatings used in commercial conductive thread. The nanowire-coated thread is also more mechanically flexible, with its resistance increasing only $14 \%$ after 200 bending cycles while the resistance of the commercial conductive thread increased by more than $300 \%$. The ability of the thread to function as a heater was demonstrated. A stretchable thread was also coated with nanowires and it was shown that its resistance converged to a stable value after several stretching cycles.

Silver nanoparticles already exist in commercially available consumer products, including textiles. Fortunately, silver nanowires have low cytotoxicity (toxicity to cells), and in the form of a connected film their toxicity is even less. ${ }^{33}$ However, as with any nanomaterial, their health and environmental effects should be considered including their impact during their manufacture and disposal.

Overall, this work demonstrates a simple, economical, and functional conductive thread for e-textiles. Furthermore, these nanowire coated threads may enable additional applications. For example, because silver nanoparticles have antimicrobial properties, ${ }^{34}$ these threads could be used in antibacterial dressings and clothing. And reports have also used silver nanowires in biosensors, ${ }^{35,36}$ which opens the possibility of textile-integrated sensors.

\section{References}

1 M. Stoppa and A. Chiolerio, Sensors, 2014, 14, 11957.

2 A. Das, J. Krishnasamy, R. Alagirusamy and A. Basu, Fibers Polym., 2014, 15, 169.

3 R. Salvado, C. Loss, R. Gonçalves and P. Pinho, Sensors, 2012, 12, 15841.

4 P. Rai, S. Oh, P. Shyamkumar, M. Ramasamy, R. E. Harbaugh and V. K. Varadan, J. Electrochem. Soc., 2013, 161, B3116.

5 T. Chen, L. Qiu, Z. Yang and H. Peng, Chem. Soc. Rev., 2013, 42, 5031.

6 S. L. P. Tang and G. K. Stylios, Int. J. Cloth. Sci. Tech., 2006, 18, 108.

7 S. Tsukada, H. Nakashima and K. Torimitsu, PLoS One, 2012, 7, e33689.
8 Y. A. Samad, Y. Li, S. M. Alhassan and K. Liao, $R S C A d v$, 2014, 4, 16935.

9 H. Cheng, Z. Dong, C. Hu, Y. Zhao, Y. Hu, L. Qu, N. Chen and L. Dai, Nanoscale, 2013, 5, 3428.

10 K. Kawano, R. Pacios, D. Poplavskyy, J. Nelson, D. D. C. Bradley and J. R. Durrant, Sol. Energy Mater. Sol. Cells, 2006, 90, 3520.

11 D. Knittel and E. Schollmeyer, Synth. Met., 2009, 159, 1433.

12 K. Nagashio, T. Nishimura, K. Kita and A. Toriumi, Jpn. J. Appl. Phys., 2010, 1, 1.

13 L. An, X. Yang and C. Chang, IJTAN, 2013, 1, 30.

14 P. Xue, K. H. Park, X. M. Tao, W. Chen and X. Y. Cheng, Compos. Struct., 2007, 78, 271.

15 A. S. Barnard, Phys. Chem. Chem. Phys., 2012, 14, 10080.

16 A. R. Murray, E. Kisin, S. S. Leonard, S. H. Young, C. Kommineni, V. E. Kagan, V. Castranova and A. A. Shvedova, Toxicology, 2009, 257, 161.

17 H. Dong, D. Wang, G. Sun and J. P. Hinestroza, Chem. Mater., 2008, 20, 6627.

18 H. Liu, W.-P. Goh and T. B. Norsten, J. Mol. Eng. Mater., 2013, 1, 1250001.

19 B. H. Dong and J. P. Hinestroza, ACS Appl. Mater. Interfaces, 2009, 1, 797.

20 D. T. Schoen, A. P. Schoen, L. Hu, H. S. Kim, S. C. Heilshorn and Y. Cui, Nano Lett., 2010, 10, 3628.

21 A. R. Madaria, A. Kumar and C. Zhou, Nanotechnology, 2011, 22, 245201.

22 Y. Sun, B. Mayers, T. Herricks and Y. Xia, Nano Lett., 2003, 3, 955.

23 Y. L. Hsieh, A. Miller and J. Thompson, Text. Res. J., 1996, 66, 1.

24 T. Tokuno, M. Nogi, M. Karakawa, J. Jiu, T. T. Nge, Y. Aso and K. Sugarnuma, Nano Res., 2011, 4, 1215.

25 J.-Y. Lee, S. T. Connor, Y. Cui and P. Peumans, Nano Lett., 2008, 8, 689.

26 A. R. Rathmell, S. M. Bergin, Y.-L. Hua, Z.-Y. Li and B. J. Wiley, Adv. Mater., 2010, 22, 3558.

27 M. Hamedi, R. Forchheimer and O. Inganäs, Nat. Mater., 2007, 6, 357.

28 L. L. Mishnaevsky and D. Gross, Appl. Mech. Rev., 2005, 58, 338.

29 C.-H. Liu and X. Yu, Nanoscale Res. Lett., 2011, 6, 75.

30 D. A. Smith, V. C. Holmberg and B. A. Korgel, ACS Nano, 2010, 4, 2356.

31 Y. Yue, P. Liu, Z. Zhang, Z. Han and E. Ma, Nano Lett., 2011, 11, 3151.

32 F. Zu and Y. Zhu, Adv. Mater., 2012, 24, 5117.

33 N. K. Verma, J. Conroy, P. E. Lyons, J. Coleman, M. P. O'Sullivan, H. Kornfield, D. Kelleher and Y. Volkov, Toxicol. Appl. Pharmacol., 2012, 264, 451.

34 J. S. Kim, E. Kuk, K. N. Yu, J.-H. Kim, S. J. Park, H. J. Lee, S. H. Kim, Y. K. Park, Y. H. Park, C.-Y. Hwang, Y.-K. Kim, Y.-S. Lee, D. H. Jeong and M.-H. Cho, Nanomedicine, 2007, $3,95$.

35 J. Kong, A. R. Ferhan, X. Chen, L. Zhang and N. Balasubramanian, Anal. Chem., 2008, 80, 7213.

36 M.-J. Song, S. W. Hwang and D. Whang, J. Appl. Electrochem., 2010, 40, 2099. 